\title{
ANÁLISE DO COMPORTAMENTO ELETROMIOGRÁFICO E DA FORÇA DURANTE A FADIGA DO MUSCULO BÍCEPS BRAQUIAL
}

\section{ANALYSIS OF THE ELECTROMYOGRAPHIC BEHAVIOR AND STRENGTH DURING THE FATIGUE OF THE BICEPS BRACHII MUSCLE}

\author{
Gabriela Aparecida da Silveira Souza ${ }^{1}$ \\ Humberto Gimenes Macedo² \\ Virginia Klausner ${ }^{3}$ \\ Marina Vedelago Cezarini ${ }^{4}$ \\ Sergio Luiz Lemos ${ }^{5}$ \\ Alexandre Alves do Nascimento Filho ${ }^{6}$ \\ Marina Corrêa ${ }^{7}$ \\ Bruna Spinelli 8 \\ Douglas Barbaroto 9 \\ Ana Paula Pinto ${ }^{10}$ \\ Mario Lima ${ }^{11}$ \\ Rodrigo Lopes-Martins ${ }^{12}$ \\ Fernanda Púpio Silva Lima ${ }^{13}$
}

Resumo: A fadiga muscular é definida como a incapacidade de manter a contração muscular e é ocasionada por alterações bioquímicas que modificam a mecânica da contração muscular, resultando em redução da performance atlética. $\mathrm{O}$ objetivo deste estudo foi avaliar o comportamento mioelétrico e a força de indivíduos hígidos durante a fadiga do músculo bíceps braquial. O estudo foi composto por 13 voluntários do sexo masculino com idade entre 20 e 30 anos $(25 \pm 3,7)$. Para a indução da fadiga muscular foram realizadas três Contrações Isométricas Voluntárias Máximas (CIVM) com duração de 50 segundos e intervalo de 50 segundos, utilizando um dinamômetro computadorizado acoplado ao eletromiógrafo

\footnotetext{
1 Mestrado em Engenharia Biomédica pela Universidade do Vale do Paraíba (Univap), E-mail: gabrielasouza507@yahoo.com.br.

2 Graduação em Engenharia da Computação pela Universidade do Vale do Paraíba (Univap), E-mail: gimeneshumberto@outlook.com.

${ }^{3}$ Coordenadora do Curso de Engenharia Elétrica da Universidade do Vale do Paraíba (Univap), Email: virginia@univap.br.

4 Graduanda em Engenharia Elétrica pela Universidade do Vale do Paraíba (Univap), E-mail: marina.cezarini@gmail.com.

5 Mestre em Engenharia Biomédica pela Universidade do Vale do Paraíba (Univap), E-mail: lemossl@uol.com.br.

6 Graduando em Fisioterapia pela Universidade do Vale do Paraíba (Univap), E-mail: alexandrealvesnf@gmail.com.

7 Graduanda em Fisioterapia pela Universidade do Vale do Paraíba (Univap), E-mail: marinaramalho05@gmail.com.

${ }^{8}$ Mestranda em Engenharia Biomédica pela Universidade do Vale do Paraíba (Univap), E-mail: brunamoreira@hotmail.com.

${ }^{9}$ Mestrando em Engenharia Biomédica pela Universidade do Vale do Paraíba (Univap), E-mail: barbaroto@hotmail.com.

${ }^{10}$ Fisioterapeuta pela Universidade do Vale do Paraíba (Univap), E-mail: apaula@outlook.com.br.

11 Laboratório de Engenharia de Reabilitação Sensório Motora, Universidade do Vale do Paraíba (Univap), E-mail: mol@univap.br.

12 Docente do Centro Universitário UniEvangélica, E-mail: ralopesmartins@gmail.com.

${ }^{13}$ Laboratório de Engenharia de Reabilitação Sensório Motora, Universidade do Vale do Paraíba (Univap), E-mail: fpupio@univap.br.
} 
de superfície. Durante a CIVM foi avaliado o sinal eletromiográfico e a força. Foi possível observar nos resultados uma queda da força muscular e dos parâmetros avaliados por meio da eletromiografia durante a fadiga muscular. A partir da regressão linear dos dados obtidos por meio da eletromiografia e dinamometria foi possível obter o coeficiente angular da reta para cada teste (Teste 1, Teste 2 e Teste 3), nota-se que houve queda de todos os parâmetros avaliados por meio da eletromiografia de superfície e da força muscular, entretanto não houve diferença estatística entre os testes, demonstrando similaridade do comportamento do sinal entre os testes. Conclui-se, portanto, que os parâmetros eletromiográficos analisados (frequência média, frequência mediana e RMS) e a força apresentam um decréscimo durante a fadiga muscular induzida por meio da CIVM.

Palavras-chave: Eletromiografia de superfície. Dinamometria. Fadiga muscular.

Palavras-chave: Eletromiografia de superfície. Dinamometria. Fadiga muscular.

Abstract: Introduction: Muscle fatigue is defined as the inability to maintain muscle contraction and it is caused by biochemical changes that modify the mechanics of muscle contraction, resulting in reduced athletic performance. The aim of this study was to evaluate the myoelectric behavior and strength of healthy individuals during the fatigue of the biceps brachii muscle. Methods: The study was composed of 13 male volunteers aged between 20 and 30 years $(25 \pm 3.7)$. For the induction of muscle fatigue, three Maximum Voluntary Isometric Contractions (MVIC) were performed with duration of 50 seconds and a 50-second interval, using a computerized dynamometer coupled to a surface electromyograph. During MVIC, the electromyographic signal and the strength were evaluated. Results: It was possible to observe in the results a decrease in muscle strength and in the mean and median frequencies of the myoelectric signal during muscle fatigue. From the linear regression of the data obtained by electromyography and dynamometry, it was possible to obtain the slope of the line for each test (Test 1, Test 2 and Test 3). It is noted that there was a drop in all parameters evaluated by surface electromyography and muscle strength; nevertheless, there was no statistical difference between the tests, demonstrating similarity in signal behavior between the tests. Conclusions: It is concluded, therefore, that the electromyographic parameters analyzed (mean frequency, median frequency and RMS) and strength show a decrease during muscle fatigue induced by MVIC.

Key words: Surface electromyography. Dynamometer. Muscle Fatigue.

Data de submissão: 25.04.2020

Data de aprovação: 01.12.2020

Identificação e disponibilidade:

(https://revista.univap.br/index.php/revistaunivap/article/view/2503, http://dx.doi.org/10.18066/revistaunivap.v27i53.2503).

\section{INTRODUÇÃO}

A literatura descreve a fadiga muscular como a incapacidade do músculo em manter a força e potência da sua contratilidade, resultando em diminuição da força, prejuízo do controle motor, e consequentemente, levando a quadros álgicos 
(MOREIRA; TEODORO; MAGALHÃES NETO, 2008; LEAL JUNIOR et al., 2009).

A fadiga muscular precoce influencia negativamente na realização de exercícios direcionados, sejam esses exercícios realizados por pessoas saudáveis ou com alguma doença como a fibromialgia, doenças pulmonares obstrutivas crônicas, fadiga crônica, e indivíduos que apresentam quadros de espasticidade (BAUDRY et al., 2007; BACHASSON et al., 2013; GARCÍA-HERMOSOA; SAAVEDRAC; ESCALANTE, 2015; NEVES et al., 2016).

Devido aos prejuízos motores desencadeados pela fadiga muscular, diversos estudos são realizados com o intuito de melhorar compreensão e interpretação desse fenômeno e suas manifestações fisiológicas, bioquímicas e moleculares. Os métodos mais utilizados para a análise da fadiga são os marcadores bioquímicos como o lactato sanguíneo e a Proteína C Reativa (PCR), a Eletromiografia de Superfície (EMGs) e análise de força muscular (GEROLD et al., 2019; LEALJUNIOR et al., 2009).

A eletromiografia de superfície é um recurso amplamente utilizado nas áreas acadêmica e clínica para a melhor compreensão de eventos musculoesqueléticos. Entretanto, a interpretação dos sinais eletromiográficos é complexa e requer o processamento e a seleção correta dos parâmetros do sinal eletromiográfico (QUESADA et al., 2014; VASSÃO et al., 2015). Neste contexto, o objetivo deste estudo foi avaliar o comportamento mioelétrico e a força de indivíduos hígidos durante a fadiga do músculo bíceps braquial.

\section{METODOLOGIA}

O estudo foi constituído por 13 voluntários hígidos do sexo masculino com idade entre 20 e 30 anos, sendo previamente aprovado pelo Comitê de Ética e Pesquisa (CEP) da Universidade do Vale do Paraíba (CAAE 94812618.8.0000.5503) e registrado no ClinicalTrials.gov (NCT03753984). Todos os participantes concordaram e assinaram o Termo de Consentimento Livre e Esclarecido (Apêndice A).

A coleta e a interpretação dos dados foram realizadas no Laboratório de Engenharia de Reabilitação Sensório Motora (LERSM), pertencente ao Instituto de Pesquisa \& Desenvolvimento (IP\&D) da Universidade do Vale do Paraíba (Brasil). E para o processamento dos sinais de eletromiografia e dinamometria, o software ELEDA (do inglês Electromyography and Dynamometer Data Analysis) foi desenvolvido por H. G. Macedo e V. Klausner do Laboratório de Imagens e Sinais (LIS/UNIVAP) que compõe o nosso grupo de pesquisa. O ELEDA possibilita a análise simultânea dos dados obtidos por meio da eletromiografia de superfície e pela dinamometria. A Figura 1 e a Figura 2 demonstram respectivamente o modo como é visualizada a interface gráfica do ELEDA quando é plotado o sinal de EMG e dinamometria. 
Figura 1 - Interface gráfica do ELEDA com os sinais de Eletromiografia de Superfície.

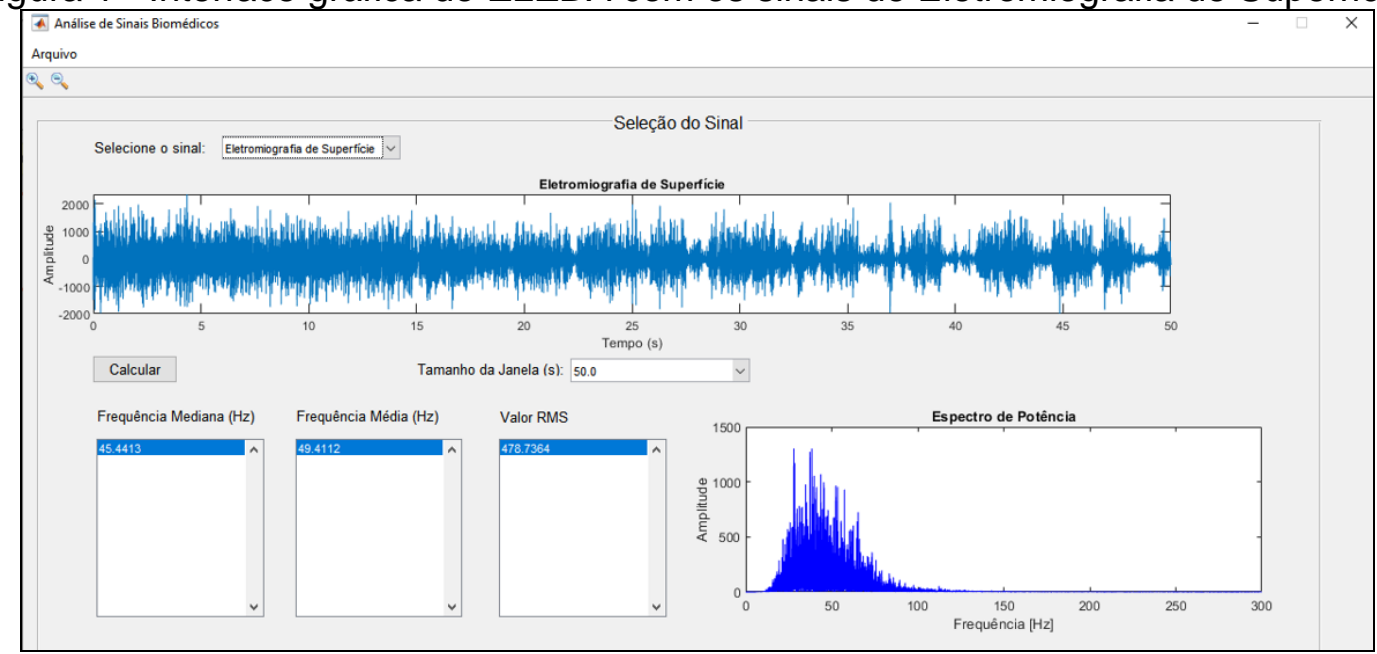

Fonte: Os autores.

Figura 2 - Interface gráfica do ELEDA com os dados de dinamometria.

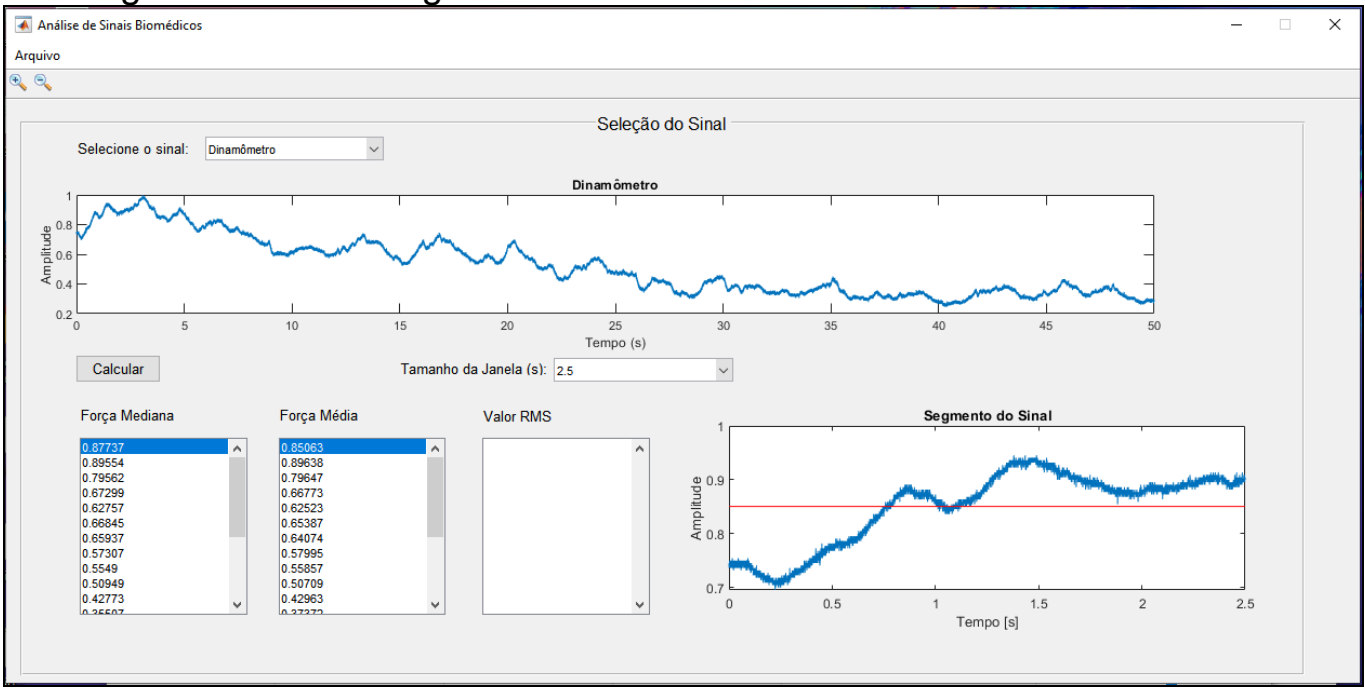

Fonte: Os autores.

Foram adotados como critérios de inclusão indivíduos hígidos, do sexo masculino, com idade entre 20 e 30 anos e que possuíssem cognição preservada com capacidade de responder a estímulos verbais. Foram adotados como critérios de exclusão indivíduos com comprometimento musculoesquelético do membro superior dominante, que praticassem atividade física com carga (musculação), apresentassem infecção ativa e erupções no membro superior dominante ou dor limitante que impossibilite a realização do protocolo de avaliação.

\subsection{MATERIAIS DE AVALIAÇÃO}

\subsubsection{Eletromiografia de superfície (EMGs)}

Os sinais mioelétricos foram coletados por um eletromiógrafo composto por 8 canais da marca EMG System Brasil Ltda, modelo EMG 832C - WIFI, composto por um conversor $A / D$ (conversor analógico-digital) de 12 bits de resolução, com frequência de amostragem de $2.000 \mathrm{~Hz}$, unidade $\mu v$, Xmin -2000, Ymáx 2000, Coef. A 0 e Coef. B 500, acoplado a um computador. 
Os eletrodos de superfície foram colocados aos pares sobre o ventre muscular do músculo bíceps braquial, acompanhando o sentido longitudinal das fibras musculares. A localização do ventre muscular seguiu às recomendações da SENIAM (Surface ElectromyoGraphy for the Non-Invasive Assessment of Muscles). Um elétrodo do tipo pinça untado com gel foi posicionado no processo estiloide da ulna do membro superior contralateral ao membro avaliado (Figura 01).

\subsubsection{Dinamometria}

Associado à captação do EMGs, foi realizada a mensuração da força muscular a partir da CIVM do músculo bíceps braquial, para a qual foi utilizado um dinamômetro computadorizado portátil da marca EMG System do Brasil, modelo DFE021115/200, conectado ao eletromiógrafo e este a rede de computador. Os sinais foram coletados utilizando-se uma taxa de amostragem de $2.000 \mathrm{~Hz}$.

\subsection{PROTOCOLO DE INDUÇÃO DA FADIGA MUSCULAR}

Para a realização do protocolo de indução de fadiga muscular do músculo bíceps braquial o indivíduo foi posicionado no banco Scott com o cotovelo a $45^{\circ}$. Uma faixa inelástica teve uma de suas extremidades fixa no dinamômetro computadorizado e a outra extremidade posicionada no antebraço do voluntário para realização do protocolo de CIVM (Contração Isométrica Voluntária Máxima) do músculo bíceps braquial (Figura 03).

O protocolo consistiu em três CIVM durante 50 segundos ininterruptos, com intervalo de 50 segundos entre cada contração, chamadas de Teste 1, Teste 2 e Teste 3. Durante as contrações musculares a atividade mioelétrica e força muscular foram coletadas de modo sincrônico. Utilizou-se como critério para considerar a fadiga muscular uma queda maior que $20 \%$ da força muscular (BARTUZI; ROMANLIU, 2014). 
Figura 3 - Posicionamento do voluntário em sedestação no banco Scott com o membro superior a ser avaliado posicionado no dinamômetro computadorizado durante o protocolo de indução da fadiga muscular por meio da contração isométrica voluntária máxima do músculo bíceps braquial.

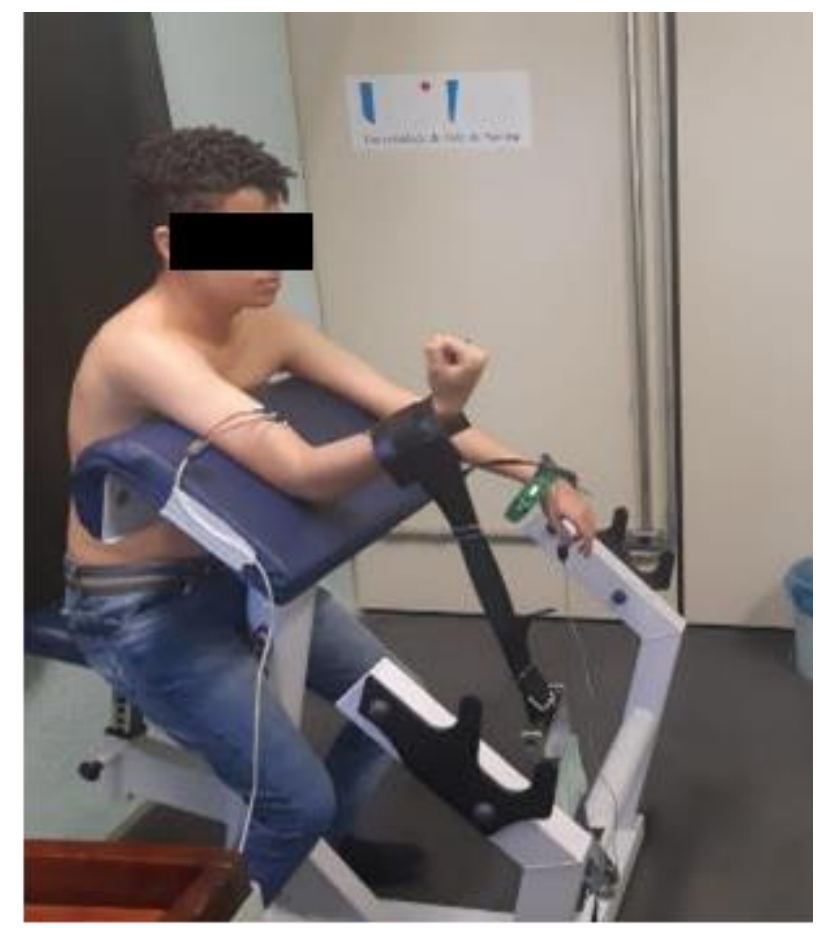

Fonte: Os autores.

\subsection{ANÁLISE DOS DADOS DA ELETROMIOGRAFIA DE SUPERFÍCIE E DINAMOMETRIA}

A EMG e a dinamometria foram realizadas de modo sincrônico e pelo mesmo equipamento, o que possibilitou a realização da análise simultânea dos sinais. Neste estudo o tempo de coleta foi de 50s para cada teste, e para o processamento do sinal foi realizado o janelamento do sinal a cada $2,5 \mathrm{~s}$ (20 janelas). Para a análise dos sinais de eletromiografia, o software faz a transformada de Fourier por meio do algoritmo da transformada rápida de Fourier (do inglês Fast Fourier Transform FFT), obtendo-se a frequência média e mediana dos sinais; e os valores de RMS (Root Mean Square) que foram obtidos por meio da amplitude do sinal temporal. Ao selecionar a análise dos sinais de dinamômetro realizou-se o cálculo da média da força em função do tempo.

O software ELEDA calculou a regressão linear a partir dos valores de saída da frequência média, frequência mediana e RMS de EMG, e média da força obtidos pela dinamometria. Posteriormente, a partir da regressão linear foi calculado os valores de coeficiente angular.

Ao selecionar a análise dos sinais de dinamômetro faz o cálculo da média em função do tempo. Para considerar fadiga muscular utilizou-se como critério a queda acima de $20 \%$ do índice de força (BARTUZI; ROMAN-LIU, 2014) calculado por meio do janelamento $\left(\frac{\text { Janela20 }}{\text { Janela } 1} \times \mathbf{1 0 0}\right)$, os voluntários que não atingiram queda acima de $20 \%$ foram excluídos do estudo.

\subsection{ANÁLISE ESTATÍSTICA}


Realizou-se a análise estatística dos dados no programa Past@ versão 4.0. Para análise descritiva utilizou-se a tendência central e o desvio padrão (DP). Todos os dados passaram pelo Teste de Normalidade Shapiro-Wilk, para as amostras consideradas normais utilizou-se o teste estatístico $t$-student, e para as amostras consideradas não-normais utilizou-se o teste Wilcoxon. $\mathrm{O}$ alfa foi ajustado para um nível de significância de $p<0,05$.

\section{RESULTADOS}

A Tabela 1 representa a caracterização da amostra do estudo.

Tabela 1 - Caracterização da amostra em relação a idade, altura, peso e índice de massa corporal.

\begin{tabular}{cc}
\hline Variáveis & Média \pm Desvio padrão \\
Idade & $25 \pm 3,7$ anos \\
Altura & $174 \pm 6,52 \mathrm{~cm}$ \\
Peso & $78 \pm 16 \mathrm{Kg}$ \\
Índice de massa corporal (IMC) & $25 \pm 4,2 \mathrm{Kg} / \mathrm{m}^{2}$ \\
\hline
\end{tabular}

Fonte: Os autores.

A seguir serão apresentados os gráficos da regressão linear dos dados da eletromiografia de superfície (frequência média, frequência mediana e valor de RMS) e da dinamometria (força média) obtidos pelo Software ELEDA.

A Figura 4 apresenta os valores de frequência média e mediana obtidos por meio da análise dos sinais de eletromiografia de superfície do músculo bíceps braquial, na qual mostra o Teste 1 (Figura 4-A), o Teste 2 (Figura 4-B) e o Teste 3 (Figura 4-C).

Nos resultados apresentados em relação às frequências dos sinais de eletromiografia, é possível observar um decréscimo dos valores das frequências média e mediana durante a fadiga muscular. 
Figura 4 - Regressão linear dos valores da frequência média e mediana obtidos pelo Software ELEDA.

A)

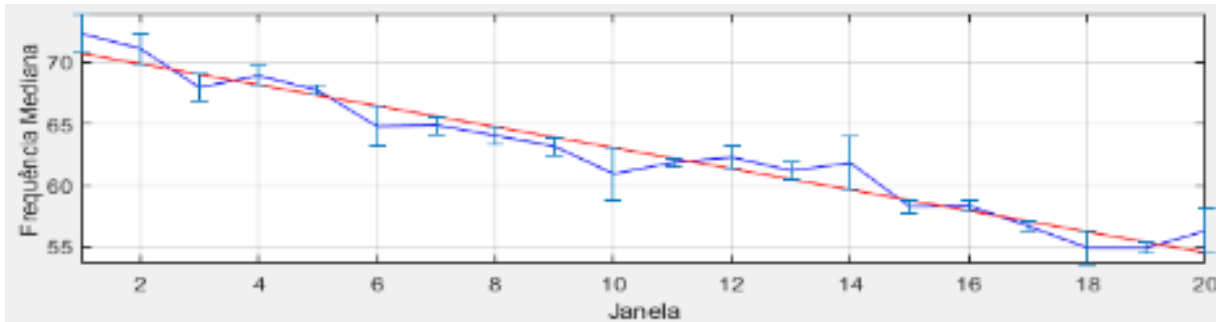

$y=71.603-0.855 x$

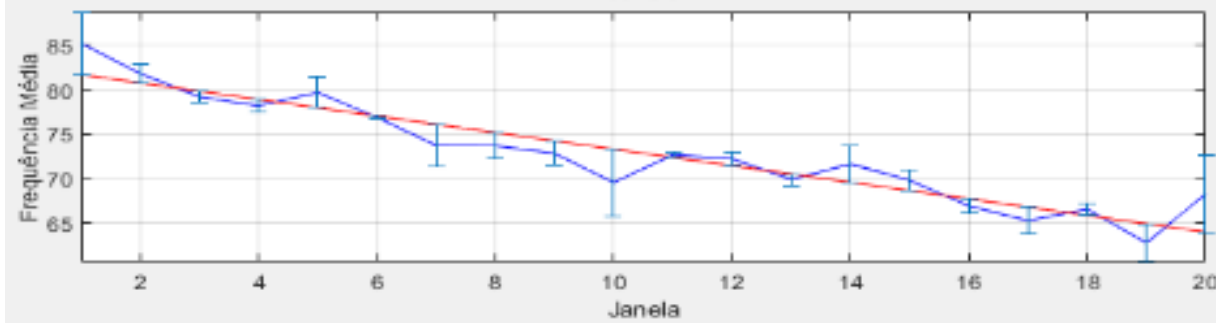

$y=82.625-0.932 x$

B)

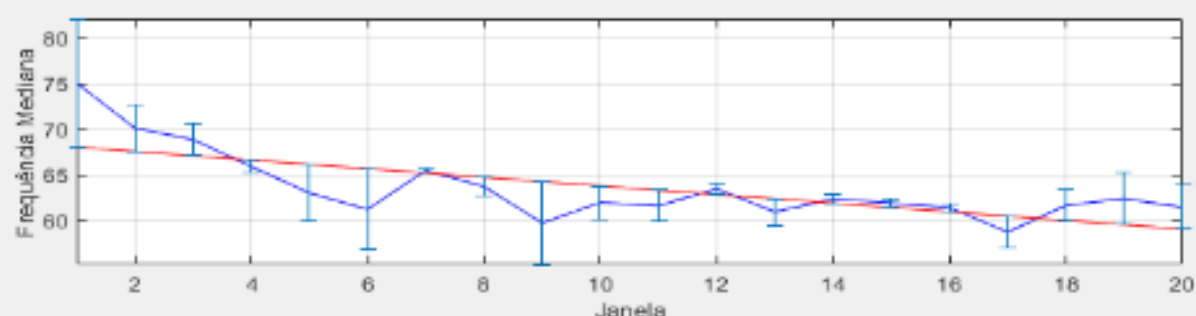

$y=68.505-0.472 x$

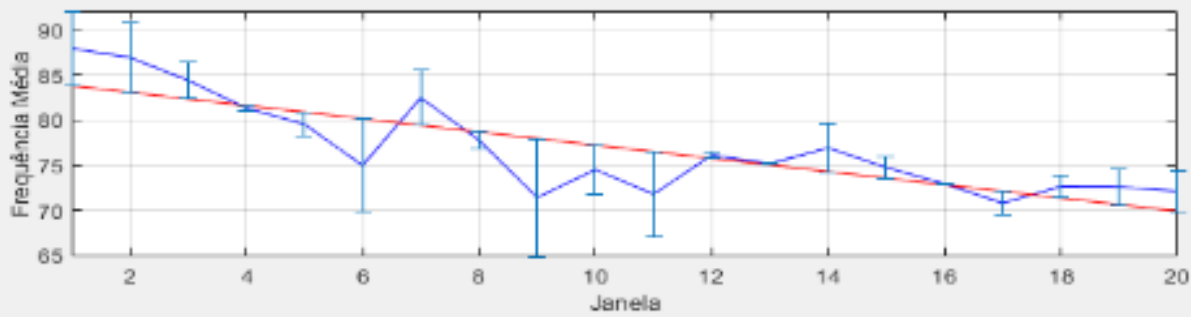

$y=64.540-0.731 x$

C)

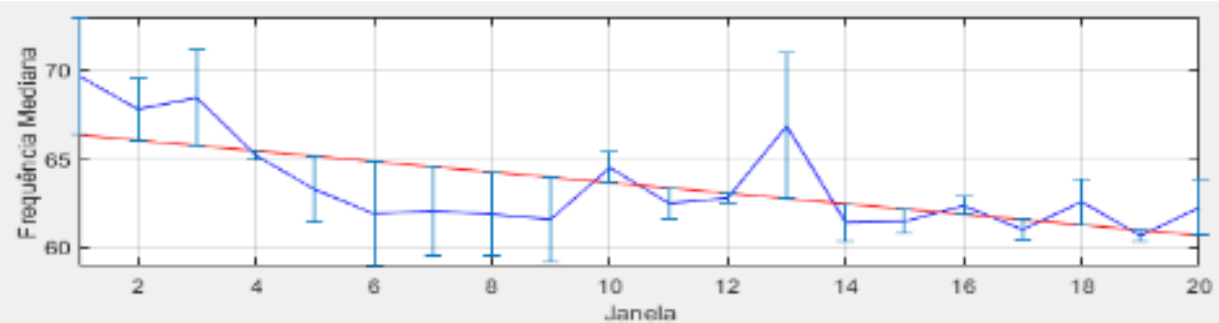

$y=66.630-0.290 x$

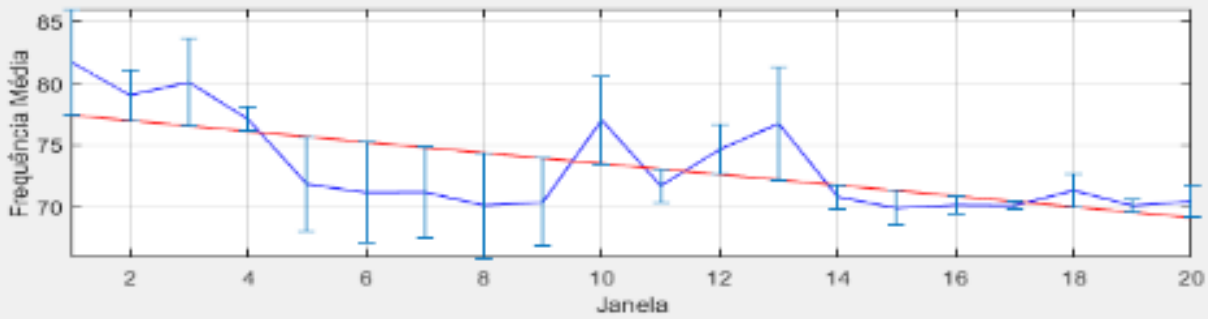

$y=77.864-0.436 x$

Legenda: Regressão linear do Teste1 (Figura 4-A); Regressão linear do Teste 2 (Figura 4-B); Regressão linear do Teste 3 (Figura 4-C). A linha azul representa a média dos valores de todos voluntários considerando janelamento de $2,5 \mathrm{~s}$, e seu respectivo erro em relação a reta de regressão (cor vermelha).

Fonte: Os autores.

A Figura 5 apresenta os valores de RMS obtidos por meio da análise temporal 
dos sinais de eletromiografia de superfície. Pode-se observar uma diminuição crescente desses valores no Teste 1(Figura 5-A), Teste 2 (Figura 5-B) e Teste 3 (Figura 5-C).

Figura 5 - Regressão linear dos valores de Root Mean Square obtidos pelo Software ELEDA.

A)

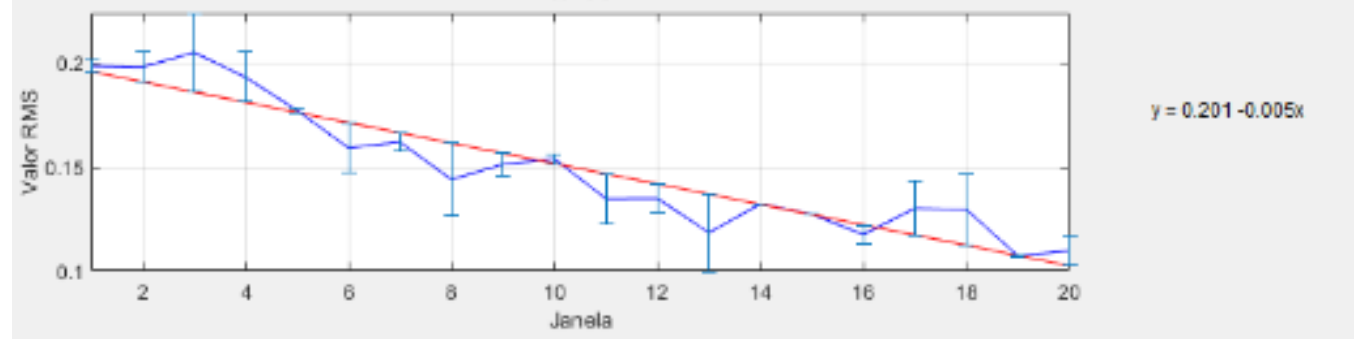

B)

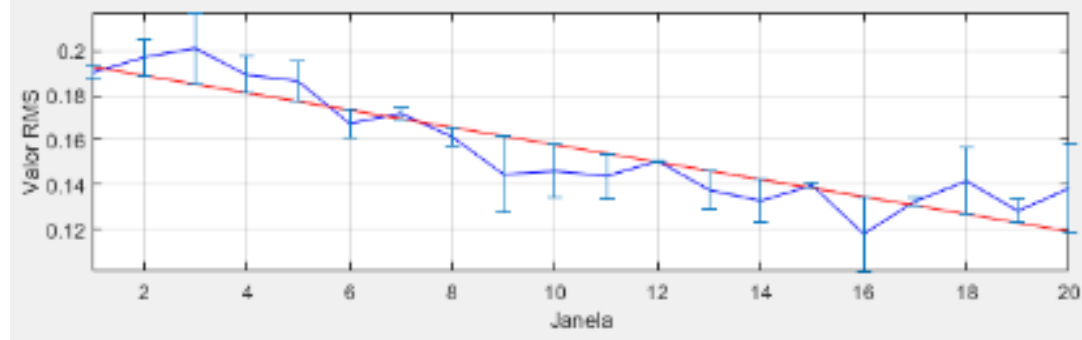

$y=0.197-0.004 x$

C)

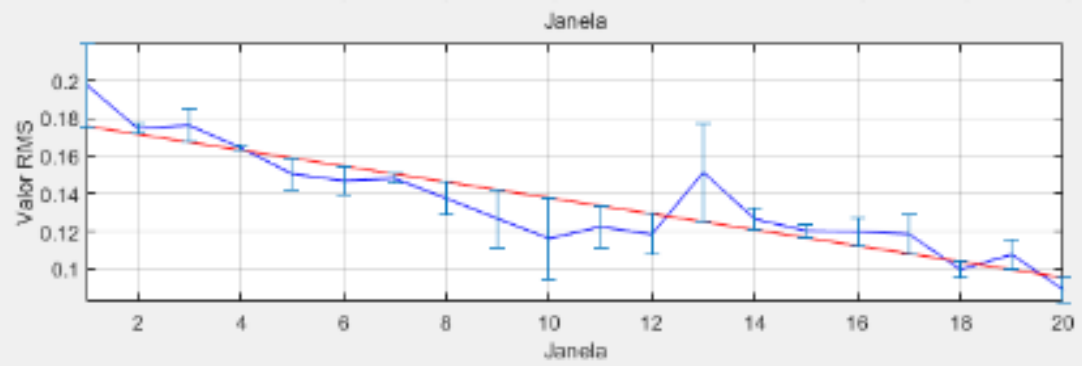

$y=0.180-0.004 x$

Legenda: Regressão linear do Teste1; (Figura 5-A); Regressão linear do Teste 2 (Figura 5-B); Regressão linear do Teste 3 (Figura 5-C). A linha azul representa a média dos valores de todos voluntários considerando janelamento de 2,5s, e a linha vermelha representa a regressão linear desses valores.

Fonte: Os autores. 
É representada na Figura 6 a força muscular durante o protocolo de indução da fadiga muscular, para o Teste 1 (Figura 6-A), o Teste 2 (Figura 6-B) e o Teste 3 (Figura 6-C).

Figura 6 - Regressão linear dos valores da força média obtidos pelo Software ELEDA.

A)

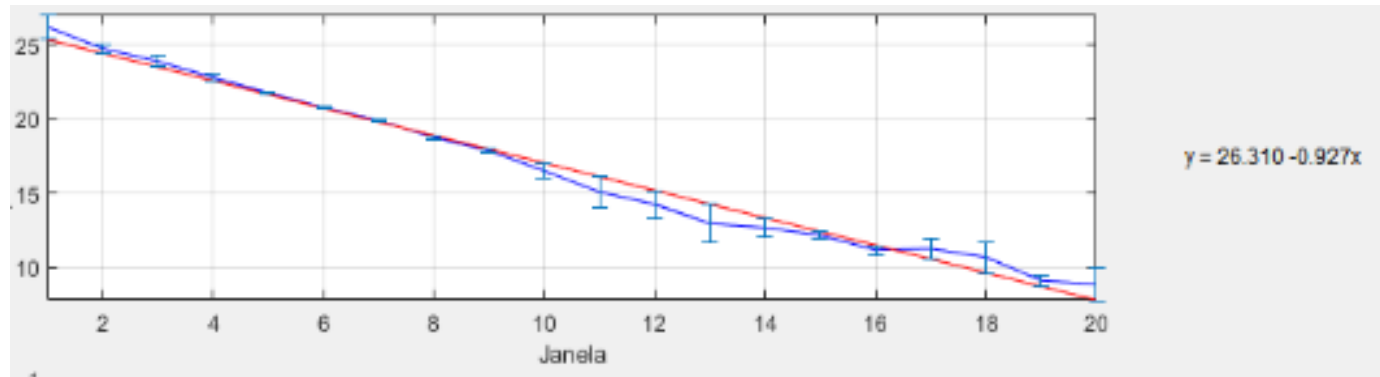

B)

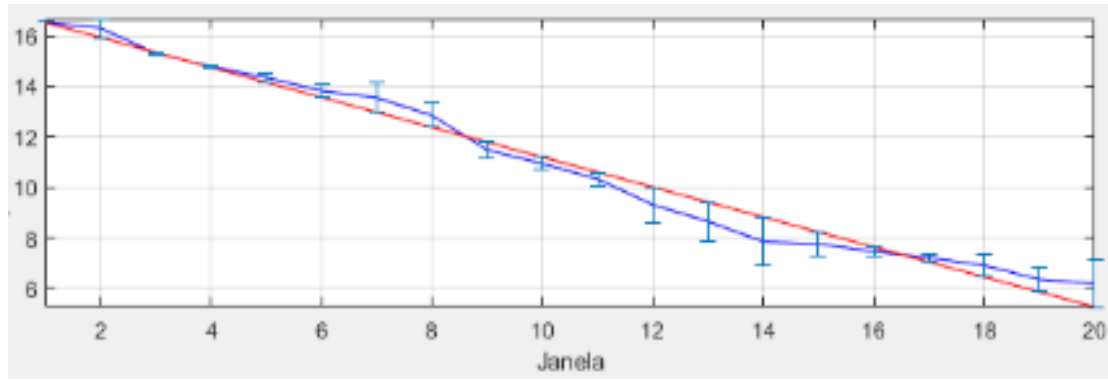

C)

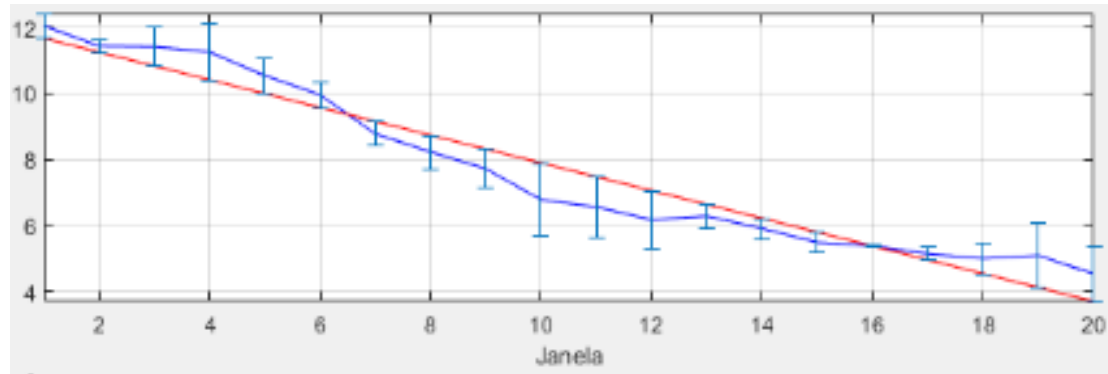

$y=12.092-0.419 x$

Legenda: Regressão linear do Teste1; (Figura 6-A); Regressão linear do Teste 2 (Figura 6-B); Regressão linear do Teste 3 (Figura $6-\mathrm{C}$ ). A linha azul representa a média da força obtidos dos voluntários por janelamento, e a linha vermelha representa a regressão linear dos valores obtidos por janelamento.

Fonte: Os autores.

Por meio da metodologia adotada para a análise dos sinais, tornou-se possível obter o coeficiente angular da reta utilizando regressão linear. A Tabela 2 expressa os valores de $p$, obtidos por meio da comparação dos testes (Teste $1 \times$ Teste 2; Teste 1 X Teste 3; Teste $2 \times$ Teste 3). Quanto mais negativo for o coeficiente angular maior será o ângulo de inclinação da reta, ou seja, mais acentuado será o decréscimo da frequência e/ou força. Considera-se também que quanto menos negativo for o coeficiente angular e próximos do valor zero, menor será o ângulo de inclinação da reta, ou seja, maior foi o tempo para ocorrer a fadiga muscular. 
Tabela 2 - Resultado da análise estatística do coeficiente angular dos parâmetros de eletromiografia de superfície do músculo bíceps braquial (frequência mediana, frequência média e RMS) e do coeficiente angular da força muscular obtida por meio do dinamômetro computadorizado do músculo bíceps braquial.

\begin{tabular}{|c|c|c|c|}
\hline Parâmetro & $\begin{array}{c}\text { Coeficiente } \\
\text { angular } \\
\text { Teste } 1 X \text { Teste 2 }\end{array}$ & $\begin{array}{c}\text { Coeficiente } \\
\text { angular } \\
\text { Teste } 1 \times \text { Teste } 3\end{array}$ & $\begin{array}{c}\text { Coeficiente } \\
\text { angular }\end{array}$ \\
\hline $\begin{array}{l}\text { Frequência mediana } \\
\qquad \text { (EMG) }\end{array}$ & $p>0,05$ & $p<0,05$ & $p>0,05$ \\
\hline $\begin{array}{l}\text { Frequência média } \\
\qquad(\text { EMG) }\end{array}$ & $p>0,05$ & $p>0,05$ & $p>0,05$ \\
\hline RMS (EMG) & $p>0,05$ & $p>0,05$ & $p>0,05$ \\
\hline $\begin{array}{c}\text { Força média } \\
\text { (Dinamometria) }\end{array}$ & $p>0,05$ & $p<0,05$ & $p>0,05$ \\
\hline
\end{tabular}

Fonte: Os autores.

Ao observar os resultados apresentados na Tabela 2 , nota-se que apenas o a frequência mediana e a força apresentaram diferença significativa entre o Teste $1 \mathrm{e}$ Teste $3(p<0,05)$, entretanto os outros parâmetros não apresentaram diferenças significativas $(p>0,05)$. Deste modo nota-se que todos os parâmetros avaliados apresentaram queda, entretanto o comportamento do sinal foi similar ao comparar os testes.

\section{DISCUSSÃO}

Para a mensuração da resposta muscular do bíceps braquial à fadiga induzida, diversos métodos podem ser utilizados para sua melhor compreensão, dentre eles a eletromiografia de superfície associada ao dinamômetro computadorizado, são considerados padrão-ouro dentro da Engenharia Biomédica no que se refere à instrumentação e análise de sinais (CONTESSA; ADAM; DE LUCA, 2009; ALMULLA; SEPULVEDA; COLLEY; 2011).

O intuito deste estudo foi analisar a fadiga em relação ao tempo, considerando que o nível de fadiga muscular dos sujeitos aumenta gradativamente até o fim do experimento. Isso pode ser comprovado pela observação da diminuição gradativa da força produzida pelos indivíduos, como evidenciado na Figura 6. Fato que corrobora com os achados de Oranchuk e colaboradores (2019), o qual demonstra o decréscimo da força no protocolo de indução de fadiga de 60 segundos de CIVM.

A fadiga muscular é definida como uma falha ou incapacidade de manutenção do mesmo nível de rendimento da força muscular durante uma determinada atividade (MATHUR; ENG; MACINTYRE, 2005). O processo de fadiga está relacionado a eventos físicos e bioquímicos, que comprometem o desempenho muscular, por exemplo o acúmulo de $\mathrm{K}$ + no sistema túbulo $\mathrm{T}$ (ALLEN; LAMB; WESTERBLAD, 2008). Outro tipo principal de fadiga são os efeitos diretos ou indiretos do acúmulo de metabólitos, como fosfato inorgânico (Pi), ADP, íons magnésio $(\mathrm{Mg}++)$, espécies reativas de oxigênio (ROS) e fosfocreatina formados a partir do quadro de fadiga (LEAL JUNIOR et al., 2009; WESTERBLAD; ALLEN, 2011; 
FERRARESI; HAMBLIN; PARIZOTTO, 2012). As ROS e espécies reativas de nitrogênio (RNS), são metabólitos formados em quadros de fadiga que podem alterar a estrutura das proteínas contráteis, diminuir a sensibilidade das miofibrilas ao $\mathrm{Ca++}$, oxidar actina, miosina e troponina $\mathrm{C}$, prejudicando a formação de pontes cruzadas, influenciando negativamente na contração muscular e produção de força muscular, esse fato é evidenciado pela diminuição da força muscular observada na dinamometria (FERRARESI et al., 2015).

Os parâmetros mais utilizados na EMG para avaliação da fadiga muscular são o RMS e as frequências do sinal (KUNISZYK-JÓŹKOWIAK; JASZCZUK; CZAPLICKI, 2018). A amplitude do sinal de EMG, expressa pelo RMS, fornece informações sobre ativação muscular, que inclui as taxas de queima e o número de unidades motoras ativadas. Foi sugerido que o domínio da frequência reflete a condução da velocidade do potencial de ação das fibras musculares durante a contração (GALEN; MALEK, 2014). A média e a mediana das frequências dos sinais de EMG são medidas comuns investigadas durante a fadiga e foram frequentemente atribuídos à mudança de pH (TSCHARNER, 2002). Deste modo entende-se que o abrandamento da velocidade de condução induzida por mudanças de $\mathrm{pH}$ reduz as frequências avaliadas pela EMG de superfície.

Durante protocolos de fadiga induzida por meio de contração isométrica submáxima ocorre aumento do RMS, o qual pode ser interpretado como 0 recrutamento adicional de unidades motoras com o intuito de manter a força muscular (SMITH, 2016). Entretanto em protocolos de contração isométrica máxima voluntária, como o que foi empregado nesse estudo, ocorre uma diminuição do RMS ao comparar o início e o final do teste, episódio observado na Figura 5, esse fato pode ser explicado em decorrência de fatores biomecânicos e bioquímicos. A contração isométrica diminui o fluxo sanguíneo e subsequente a oxigenação muscular, contribuindo assim para a fadiga neuromuscular (ORANCHUK et al., 2019).

Koral e colaboradores (2020) realizaram um estudo no qual os voluntários foram submetidos a 60 segundos de CIVM dos músculos extensores de joelho, e foi mensurada a atividade mioelétrica por meio da EMG durante a CIVM, em seus resultados foi possível observar uma diminuição da força e do RMS durante o protocolo máximo de CIVM, fato que corrobora com os achados desse estudo.

Os exercícios de alta intensidade desencadeiam grandes alterações metabólicas e iônicas como, aumento da concentração de lactato muscular, $\mathrm{H}+, \mathrm{K}+\mathrm{e}$ $\mathrm{Ca++}$, os quais estão ligados ao processo de fadiga muscular (BOGDANIS, 2012). A diminuição do $\mathrm{pH}$ intracelular, causada pelo acúmulo de metabólitos, resulta na redução da excitabilidade da membrana e, como resultado, diminuiu velocidade de propagação do potencial de ação, que pode ser refletido na redução das frequências dos sinais mioelétricos (CASTROFOLIO et al., 2012). O estudo de Smith (2016) teve por objetivo avaliar o comportamento em curso temporal das alterações das respostas neuromusculares mensuradas por meio da EMG de superfície durante um treino isométrico fatigante e intermitente e foi observado em seus resultados uma queda crescente da frequência mediana, resultado também encontrado em nosso estudo, em que os voluntários apresentaram queda na frequência dos sinais (Figura 4 e 5).

A inclinação da regressão linear é um conhecido método de avaliação da fadiga eletromiográfica (CASTROFLORIO et al., 2012). Pitta e colaboradores (2015) realizaram um estudo utilizando a EMG de superfície com o objetivo de comparar o tempo relativo de ativação do músculo elevador mandibular em diferentes níveis de 
atividade e frequência média durante a mordida sustentado. Regressões lineares simples foram realizadas para descrever a relação entre a frequência média (variável dependente) e tempo de manutenção da mordida (variável independente). Coeficientes angulares (declive) foram calculados por meio equações de regressão linear que se ajustam aos valores da frequência média ao longo do tempo como um índice de fadiga.

O benefício clínico da análise das alterações musculares em indivíduos hígidos durante a fadiga muscular induzida avaliada por meio da EMG de superfície associada a dinamometria, permite a melhor interpretação fisiológica antes de sua aplicação em pacientes.

\section{CONSIDERAÇÕES FINAIS}

Os achados deste estudo demonstram que a avaliação eletromiográfica associada à dinamometria são consideradas importantes ferramentas da Engenharia Biomédica para compreender o fenômeno de fadiga muscular. Conclui-se que durante o processo de fadiga do músculo bíceps braquial em um protocolo de CIVM ocorreu uma queda de todos os sinais eletromiográfico analisados (frequência média e mediana, RMS) assim como a força muscular.

\section{AGRADECIMENTOS}

O presente trabalho foi realizado com apoio da Coordenação de Aperfeiçoamento de Pessoal de Nível Superior - Brasil (CAPES) - Código de Financiamento 001.

\section{REFERÊNCIAS}

ALLEN, D.G.; LAMB, G.D.; WESTERBLAD, H. Skeletal muscle fatigue: cellular mechanisms. Physiol Rev. v. 88, n. 1, p. 287-332, 2008.

AL-MULLA, M. R.; SEPULVEDA, F.; COLLEY, M. A Review of Non-Invasive Techniques to Detect and Predict Localised Muscle Fatigue. Sensors, v. 11, p. 35453594, 2011.

BACHASSON, D. et al. Neuromuscular Fatigue and Exercise Capacity in Fibromyalgia Syndrome. Arthritis Care Res. v. 65, n. 3, p. 432-440, 2013.

BAUDRY, S. et al. Age-related fatigability of the ankle dorsiflexor muscles during concentric and eccentric contractions. Eur J Appl Physiol. v. 100, p. 515-525, 2007.

BARTUZI, P.; ROMAN-LIU, D. Assessment of muscle load and fatigue with the usage of frequency and time-frequency analysis of the EMG signal. Acta Bioeng Biomech, v. 16; n. 2, p. 31-39, 2014.

BOGDANIS, G. C. Effects of physical activity and inactivity on muscle fatigue. Front Physiol., v. 3, p. 1-15, 2012. 
CASTROFLORIO, T. et al. Myoelectric manifestations of jaw elevator muscle fatigue and recovery in healthy and TMD subjects. J Oral Rehabil., n. 39, n. 9, p. 648-658, 2012.

CONTESSA, P.; ADAM, A.; DE LUCA, D. J. Motor unit control and force fluctuation during fatigue. J Appl Physiol., v. 107, p. 235-243, 2009.

FERRARESI, C.; HAMBLIN, M. R.; PARIZOTTO, N. A. Low-level laser (light) therapy (TLBI) on muscle tissue: performance, fatigue and repair benefited by the power of light. Photonics Lasers Med., v. 1, n. 4, p. 267-286, 2012.

FERRARESI, C. et al. Time response of increases in ATP and muscle resistance to fatigue after low-level laser therapy in mice. Lasers Med Sci., v. 30, p. 1259-1267, 2015.

GALEN, S. S.; MALEK, M. H. A single electromyographic testing point is valid to monitor neuromuscular fatigue during continuous exercise. J Strength Cond Res., v. 28, n.10, p. 2754-2759, 2014.

GARCÍA-HERMOSOA, A.; SAAVEDRAC, J. M.; ESCALANTE, Y. Effects of exercise on functional aerobic capacity in adults with fibromyalgia syndrome: A systematic review of randomized controlled trials. J Back Musculoskelet Rehabil., v. 28, n. 2015, p. 609-619, 2015.

GEROLD, E. et al. Age- and sex-specific effects in paravertebral surface electromyographic back extensor muscle fatigue in chronic low back pain. GeroScience, v. 42, n.1, p. 251-269, 2019.

KORAL, J. et al. Mechanisms of neuromuscular fatigue and recovery in unilateral versus bilateral maximal voluntary contractions. J Appl Physiol, v. 128, n. 4, p. 785794, 2020.

KUNISZYK-JÓŹKOWIAK, W.; JASZCZUK, J.; CZAPLICKI, A. Changes in electromyographic signals and skin temperature during standardised effort in volleyball players. Acta Bioeng Biomech., v. 20, n. 3, p. 115-122, 2018.

LEAL JUNIOR, E. C. P. et al. Effect of cluster multi-diode light emitting diode therapy (LEDT) on exercise-induced skeletal muscle fatigue and skeletal muscle recovery in humans. Lasers Surg Med., v. 41, n. 8, p. 572 - 577, 2009.

MATHUR, S.; ENG, J. J.; MACINTYRE, D. L. Reliability of surface EMG during sustained contractions of the quadriceps. J Electromyogr Kinesiol., v. 15, n. 2005, p. 102-110, 2005.

MOREIRA, P. V. S.; TEODORO, B. G.; MAGALHÃES NETO, A.M. Neural and metabolic bases of the fatigue during the exercise. Biosci. J., v. 24, n. 1, p. 81-90, 2008.

NEVES, M. F. et al. Effects of low-level laser therapy (TLBI $808 \mathrm{~nm}$ ) on lower limb spastic muscle activity in chronic stroke patients. Lasers Med Sci., v. 31, n. 7, p. 
1293-1300, 2016.

ORANCHUK, D. J. et al. Effect of blood flow occlusion on neuromuscular fatigue following sustained maximal isometric contraction. Appl Physiol Nutr Metab. p. 129, 2019.

PITTA, N. C. et al. Activation time analysis and electromyographic fatigue in patients with temporomandibular disorders during clenching. J Electromyogr Kinesiol., v. 25, n. 4, p. $653-657,2015$.

QUESADA, J. I. P. et al. Relationship between skin temperature and muscle activation during incremental cycle exercise. J. Therm. Biol., v. 48, p. 28-35, 2014.

SMITH, C. M. Combining regression and mean comparisons to identify the time course of changes in neuromuscular responses during the process of fatigue.

Physiol. Meas., v. 37, p. 1993-2002, 2016.

TSCHARNER, V. V. Time-frequency and principal-component methods for the analysis of EMGs recorded during a mildly fatiguing exercise on a cycle ergometer. $\mathbf{J}$ Electromyogr Kinesiol., v. 12, n. 2002, p. 479-492, 2002.

VASSÃO, P. G. et al. Effects of photobiomodulation on the fatigue level in elderly women: an isokinetic dynamometry evaluation. Lasers Med Sci., v. 31, n. 2, p. 275282, 2015.

WESTERBLAD, H.; ALLEN, D. G. Emerging roles of ROS/RNS in muscle function and fatigue. Antioxid Redox Signal, v. 15, n. 9, p. 2487-2499, 2011. 